\title{
Método de madurez para la calidad de los datos
}

Data quality maturity method

Fecha de recepción: 9 de febrero de 2012

Fecha de evaluación: 12 de abril de 2012 Fecha de aprobación: 4 de septiembre de 2012

\section{Carlos Orlando Hernández Gama (Colombia)}

chernandez@sinertic.com.co

ESI Center SinerTIC Andino

Ingeniero de Sistemas de la Universidad Politécnico Grancolombiano, Especialista en Gerencia de la Universidad Pontificia Bolivariana de Medellín; Investigador de la Universidad Externado de Colombia, Consultor especializado para la creación de técnicas y metodologías de gestión empresarial ESI Center SinerTIC Andino. Consultor y director de proyectos de tecnología en empresas del estado y privadas.

\section{Brigitte Mayorga Jaimes (Colombia)}

ESI Center SinerTIC Andino

Ingeniera de Sistemas y Computación de la Universidad de los Andes, con amplia experiencia en servicios informáticos. Gestora en el año 2003 del Clúster de Tecnologías de Información y Servicios Asociados SinerTIC, labor que la ha hecho merecedora de múltiples reconocimientos internacionales. Actualmente se desempeña como consultora nacional e internacional en modelos asociativos, consorcios de exportación de software y en la Presidencia del Centro de Desarrollo Tecnológico de Tecnologías de la Información ESI Center SinerTIC Andino

\section{Lyda Peña Paz (Colombia)}

Ipena@uao.edu.co

Universidad Autónoma de Occidente

BSc., Ingeniera de Sistemas de la Universidad de San Buenaventura Cali, Colombia, Magíster en Ciencias Computacionales del Instituto Tecnológico y de Estudios Superiores de Monterrey - ITESM, México. Estudiante del Doctorado en Ingeniería Informática - Bioinformática e Informática Biomédica, Universidad Pontificia de Salamanca, España. Docente de Planta, Universidad Autónoma de Occidente, Cali. 


\section{Resumen}

En este artículo se presenta la propuesta de un método para la valoración del nivel de madurez en la calidad de los datos (IQM-QDMM) que permite a las organizaciones identificar y fortalecer sus prácticas empresariales y la tecnología utilizada para el tratamiento de los datos, a fin de asegurar la calidad en los sistemas de información como un factor para la competitividad.

Este método se basa en el modelo de madurez IQM (por sus siglas en inglés Information Quality Metrics) propuesto por ESI Center SinerTIC Andino. IQM define las perspectivas organizacionales y los niveles de madurez para la valoración y tratamiento de los datos en la organización, a partir de lo cual, se determina un sistema de métricas, las condiciones de las áreas de valor del modelo de madurez y los instrumentos para llevar a cabo dicha valoración.

\section{Palabras clave}

Calidad de datos, modelo de madurez, gestión empresarial.

\section{Abstract}

In this article a proposal of a valuing method on maturity level in data quality (IQM-QDMM) is presented. This method allows organizations to identify and strengthen their corporate practices and the technology used for data processing to ensure the quality in the information systems understood as a key factor for competitivity.

This method is based on the maturity model IQM (Information Quality Metrics) proposed by the ESI Center SinerTIC Andino, IQM defines the organizational perspectives and the maturity levels for valuing and data processing within the organization. Based on this, a metrics system, the value area conditions of the maturity model and the instruments to perform such valoration are determined.

\section{Keywords}

data quality, maturity model, corporate management. 


\section{Introducción}

En las últimas décadas, las organizaciones se han visto enfrentadas a un mercado globalizado que las obliga a mejorar su competitividad y productividad, mejorando la calidad de sus productos y servicios basados en la optimización de los recursos, la eficiencia de sus operaciones y la calidad de su información. En este contexto, cobra aún mayor importancia el manejo que se haga de la información al interior de la organización.

$\mathrm{Si}$ bien, las organizaciones entienden la importancia de la calidad de la información y han realizado inversiones importantes en procesos de automatización, siguen encontrándose inconvenientes al momento de requerir datos precisos que apoyen la implementación de estrategias de negocio o la toma de decisiones.

Atendiendo la necesidad de valorar el nivel de calidad de los datos que se manejan en una organización, ESI Center SinerTIC Andino propuso la metodología IQM como un servicio integrado que provee soluciones para el mejoramiento de la calidad de los datos que soportan los procesos estratégicos del negocio (ESI Center, 2009).

Sustentado en la definición del servicio IQM, el presente documento plantea la propuesta de un modelo para medir el nivel de Madurez de las organizaciones en cuanto a la Calidad de los Datos (Módulo de métricas IQM-QDMM) que permita identificar y fortalecer sus prácticas empresariales y la tecnología utilizada para el tratamiento de los datos, a fin de asegurar la calidad en los sistemas de información (ESI Center - Sinertic, 2011).

A continuación se hace una descripción de la metodología IQM y se plantean los factores, elementos y niveles que considera el Modelo de Madurez para la Calidad de los Datos, posteriormente se presenta un caso de aplicación del método y sus resultados.

\section{Calidad de los datos en el contexto organizacional}

En las organizaciones, los datos se reconocen como activos valiosos y como un componente esencial para el éxito de las estrategias, no obstante, muchos sistemas arrojan pobres resultados que alejan a los clientes, porque producen altos costos de mantenimiento y reducción de la eficiencia, efectividad y rentabilidad de las empresas (ESI Center - SinerTIC, 2011).

A continuación se mencionan algunos de los inconvenientes que se presentan al interior de las organizaciones en cuanto al manejo de los datos, los cuales se reflejan en la baja calidad de los mismos.

- No se poseen mecanismos para establecer las pérdidas financieras causadas por la deficiente calidad de los datos.

- No se han definido políticas que establezcan responsabilidades y acciones enfocadas a garantizar la calidad en el manejo de los datos y la información.

- No hay cultura organizacional en lo correspondiente a la apropiación de la calidad de los datos y de la información.

- Se observa el manejo descentralizado de los datos, lo cual no garantiza consistencia e integridad, redundando en bajos resultados y sobrecostos de procesamiento. 
- Carencia de procedimientos de control y seguimiento a la calidad de los datos, por la debilidad o ausencia de indicadores de satisfacción o métricas de calidad.

- Se asocia la calidad de los datos específicamente con la gestión tecnológica y no se consideran otras perspectivas organizacionales que igualmente la afectan.

- No se aplican las mejores prácticas de ingeniería para el desarrollo y mantenimiento del software que administra los datos en su ciclo de vida.

En un estudio realizado a $2600 \mathrm{em}$ presas españolas de todos los sectores (ESIC y Schober, 2006), se muestra que aun cuando el $74 \%$ de ellas reconoce tener problemas con la calidad de los datos y manifiesta la necesidad de disponer de información más confiable para la toma de decisiones, solamente el $8,7 \%$ han implementado un plan de mejora para la calidad de sus datos, y el $25,4 \%$ están implementando un plan, el $23,2 \%$ están desarrollando un plan y el $42,7 \%$ no tienen ningún plan de mejora.
Figura 1. Proporción de empresas

que cuentan con una estrategia formal de calidad de datos

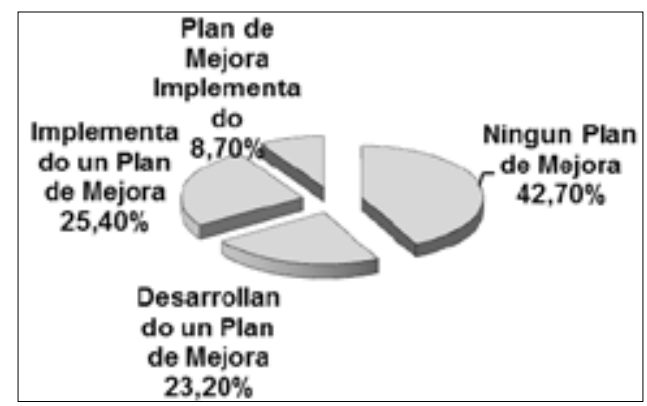

Fuente: ESIC-SCHOBER, 2006.

Considerando la importancia que tienen los datos para el éxito de las organizaciones en su entorno competitivo, una pobre calidad de datos puede tener un fuerte impacto en la salud de una compañía. Si los datos defectuosos no se identifican y corrigen a tiempo, pueden contaminar la información precisa, elevar los costos, arriesgar las relaciones con los clientes, generar pronósticos imprecisos y decisiones incorrectas (Eckerson, 2002).

El mismo estudio mencionado anteriormente (ESIC y Schober, 2006), determina las principales áreas de las empresas españolas que resultan afectadas por la calidad de datos, las cuales se presen$\tan$ en la figura 2 . 
Figura 2. Principales áreas de las empresas españolas que

resultan afectadas por una pobre calidad de datos

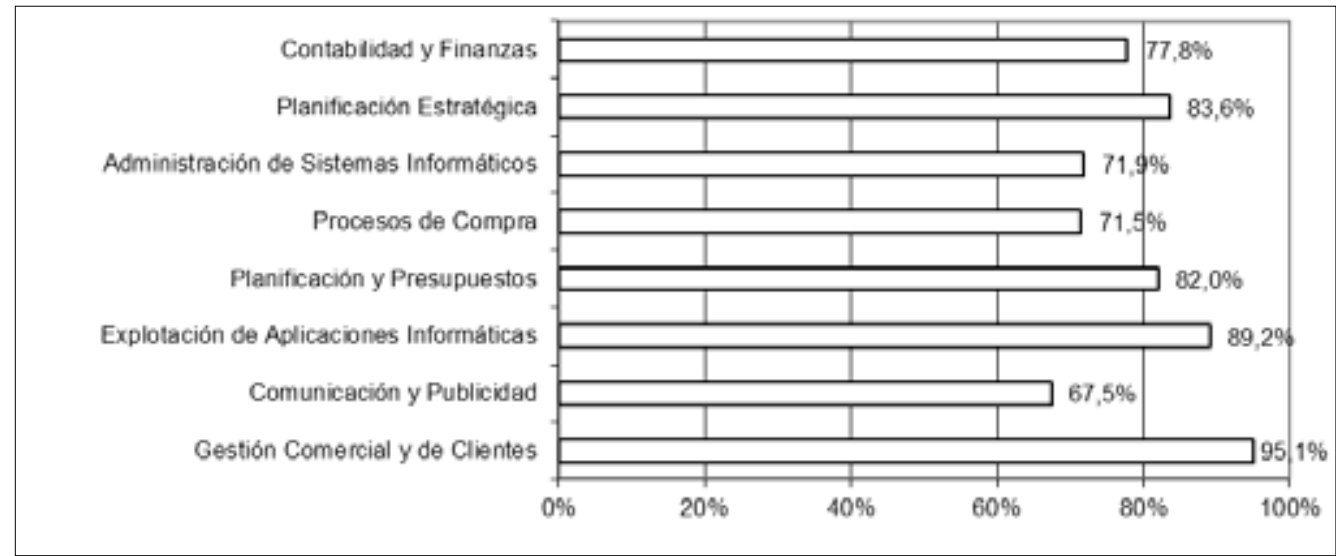

Fuente: ESIC-SCHOBER, 2006.

\section{Antecedentes}

En la revisión de literatura, se encuentran múltiples estudios y propuestas alrededor del tema de Calidad de datos, la mayor parte de los cuales se concentra en la caracterización de factores y el diseño de herramientas para asegurar un nivel aceptable de la calidad de los datos.

Algunos documentos proponen modelos para la medición de calidad de los datos; 1. (Shankar y Watts, 2003) plantean un modelo de valoración de calidad de los datos, considerando una evaluación objetivo y una evaluación contextual; 2. (Allen, Carter et al., 2003) proponen un modelo repetible para abordar la calidad de los datos cuando se desea transformar datos existentes en forma de texto a un sistema ERP (Enterprise Resource Planning); 3. (Wang y Wang, 2007) proponen un modelo de minería de datos basado en mapas auto-organizables (SOM) para visualizar los patrones de valores perdidos en una colección de datos y para valorar la calidad de los datos en términos de su completitud; 4. (Leonowich-Graham y Willshire, 2003), 5. plantean un marco de trabajo denominado SBQF (Small Business Quality Framework), basado en el modelo TDQM (Total Data Quality Mangement) para lograr que las pequeñas empresas alcancen un nivel aceptable de calidad de datos.

Entre los trabajos que se han centrado en el desarrollo de herramientas de apoyo a los procesos de calidad de datos se encuentran la propuesta de 1. (Svchenko, 2003) cuyo estudio presenta experiencias del proceso de construcción de una herramienta de software para apoyar a los analistas en la estimación objetiva de la calidad de información de una fuente de datos relacionales desconocida; 2. (Long, Seko et al., 2004) presentan una lista de chequeo preliminar de calidad de datos, basado en la teoría y prácticas del CIHI (Canadian Institute for Health Information), el cual se encuentra personalizado para facilitar las actividades de calidad de datos para recolectores de información 
primaria del servicio administrativo de salud; 3. (Kerr \& Norris, 2004) plantean una investigación que busca contribuir con el desarrollo de una estrategia de calidad de datos para el ministerio de salud de Nueva Zelanda; 4. (Oliveira, Rodrigues y Henriques, 2006) presentan una nueva alternativa para operaciones de mantenimiento de datos existentes en bases de datos, con el objetivo de automatizar dicho proceso mediante la reutilización de procesos de limpieza de datos.

En cuanto a metodologías de medición, se destacan los trabajos de 1. (Even y Shankaranarayana, 2005), quienes proponen métodos de medida para características de calidad comúnmente usadas (completitud, validez, exactitud y actualidad) basadas en el concepto de valor intrínseco; 2. (Führing y Naumann, 2007), presentan una solución para la representación gráfica de los datos con puntajes de calidad para permitir su eficiente almacenamiento y recuperación; 3. (Hipp,Müler et al., 2007) amplían y evalúan un enfoque para medición de la dimensión de exactitud de la calidad de datos basado en reglas de asociación, el modelo propuesto es empleado para dividir los registros de una base de datos en tres subconjuntos: potencialmente incorrectos, calidad no definida y probablemente correctos; 4. (Batini, Barone et al., 2007) proponen ORME-DQ que es una metodología y un marco de trabajo asociado para la valoración de la calidad de datos en las organizaciones, igualmente hacen un análisis comparativo de diferentes metodologías disponibles para valoración de calidad de datos; 5. (Heinrich, Kaiser y Klier, 2007) plantean una metodología para la cuantificación de la dimensión de disponibilidad de los datos;
6. (Amicis, Barone y Batini, 2006) presentan un marco de trabajo analítico que incluye los métodos principales de dependencia y formulación analítica, basados en la entropía de Shannon, cada modelo es caracterizado y se proponen medidas de correlación entre las dimensiones.

\section{Modelo de Madurez IQM}

Los modelos de madurez están siendo aplicados en las organizaciones como un mecanismo que busca garantizar la correcta incorporación de prácticas de gestión, para llegar a un estado ideal que les permita obtener mayor competitividad.

El modelo de madurez IQM es un modelo adoptado de las mejores prácticas de medición de las capacidades empresariales, enfocado al análisis y valoración del tratamiento y administración de los datos; examina un conjunto de prácticas y procesos que se han agrupado en cuatro dimensiones o perspectivas de la organización y sobre las cuales, se considera que deben ser incorporadas técnicas, herramientas y mecanismos para que la estructura de datos que se maneja al interior de la organización cuente con los elementos de calidad requeridos.

El modelo de Madurez IQM, se diferencia de otros modelos debido a que 1) emplea un enfoque sistémico; 2) emplea un progreso escalado en la calidad de datos; 3) formula recomendaciones para su aplicación práctica y efectiva; 4) Emplea un enfoque estratégico del modelo y lo vincula con la gestión del conocimiento y 5) se vinculan diferentes dimensiones o perspectivas de la organización; 6) propone mejoras de procesos en operación y alternativas de implementación de productos y/o metodologías que logran altos 
impactos en la efectividad operativa, técnica y financiera.

\section{Elementos que conforman \\ el modelo de madurez}

El modelo de madurez de calidad de datos se fundamenta sobre cuatro componentes: los referentes de calidad del dato, el ciclo de vida del dato, las perspectivas organizacionales y los niveles de madurez.

\section{Referentes de Calidad del Dato}

Los referentes de calidad del dato se establecen para determinar el tipo de inconsistencia que pueden presentar los datos a partir de los factores de vulnerabilidad identificados y los procesos de levantamiento y análisis de condiciones del ciclo de vida de los datos en los procesos organizacionales; estos referentes son: dato exacto, dato integral, dato consistente y dato completo.

1. El ciclo de vida del dato

Por otro lado, al contemplar la información como un producto de procesamiento de los datos se determinan cuatro estados que componen el ciclo de vida del dato: origen, administración, transformación y uso. Uno de los aspectos que valora la línea base es el nivel de identificación y estructuración que tiene la organización en la trazabilidad de los datos, la cual está determinada por la identificación del dato en la cadena de valor de la información estratégica que integra las cuatro fases del ciclo de vida del dato; desde esta premisa se puede evaluar la incidencia que tiene el ciclo de vida del dato sobre el cumplimiento de metas y competitividad de la organización.

\section{Perspectivas organizacionales para la calidad de datos}

Para esta propuesta se ha cambiado el enfoque de lo que se reconocía como áreas de conocimiento hacia una identificación de perspectivas organizacionales para la valoración de la calidad de datos.

En la metodología se afirma que la calidad de los datos se alcanza a través de la ejecución de buenas prácticas en todo el ciclo de vida del dato y para mantener este supuesto, los niveles de madurez deben definirse en términos del alcance del ciclo de vida de los datos orientados hacia su aporte a la calidad.

IQM se enfoca en los sistemas de la información estratégica, entendida como el grupo de componentes que tiene mayor impacto en la calidad de la información. Se ha contemplado que está formado por dos enfoques: los datos evaluados con los referentes de calidad definidos y las perspectivas organizacionales, que son la dirección de los procesos de su ciclo de vida, la definición y ejecución de estos, la cultura organizacional orientada a la calidad, la arquitectura y la seguridad presente. La tabla 1 describe cada una de las perspectivas en términos de la forma en la que evalúan dentro del modelo. 
Tabla 1. Perspectivas organizacionales del modelo IQM

\begin{tabular}{|l|l|}
\hline \multicolumn{2}{|c|}{ PERSPECTIVAS } \\
\hline DIRECCIÓN & $\begin{array}{l}\text { Prácticas que determinan el nivel de preparación sostenible de una organización } \\
\text { para generar información de calidad para la toma de decisiones eficientes, evi- } \\
\text { denciado a través de directrices enunciadas, oficiales, implementadas y auditadas. }\end{array}$ \\
\hline CULTURA ORGANIZACIONAL & $\begin{array}{l}\text { Es el grado de apropiación e incorporación de la calidad de los datos en la organi- } \\
\text { zación, vista a través de la difusión de las políticas asociadas y su aplicación. En el } \\
\text { proceso lQM esta perspectiva está estrechamente ligada a los factores de la pers- } \\
\text { pectiva de Dirección. }\end{array}$ \\
\hline TRAZABILIDAD & $\begin{array}{l}\text { Factores empresariales que permiten determinar el nivel de la presencia de bue- } \\
\text { nas prácticas en los procedimientos de validación, medición, seguridad y mejora- } \\
\text { miento continuo tal que se garanticen la calidad de los datos durante todo el ci- } \\
\text { clo de vida. }\end{array}$ \\
\hline ARQUITECTURA & $\begin{array}{l}\text { Es el conjunto de características de los insumos de tecnología utilizados para ge- } \\
\text { nerar la información estratégica de la organización, compuesto por la arquitectu- } \\
\text { ra de datos, de sistemas de información y la ingeniería aplicada, cuyo nivel de cali- } \\
\text { dad influye en la capacidad para asegurar la calidad del dato. }\end{array}$ \\
\hline
\end{tabular}

\section{Niveles de Madurez del Modelo IQM}

Para el modelo se establecieron tres niveles de madurez que le permite a las organizaciones trazar metas específicas para alcanzar altos estándares y buenas prácticas. Estos niveles son:

- Básico: Los sistemas de información están identificados, implementados y verificados.

- Medio: Los elementos que afectan la calidad de los datos se encuentran controlados y gestionados.

- Alto: Las actividades están estables y se aplica mejoramiento continuo.

Cada nivel tiene una serie de requerimientos para los elementos más influyentes sobre la calidad de los datos los cuales son: la información estratégica y operativa, los datos trazados, verificables y seguros, medición de la calidad de los datos, los procesos de soporte tecnológico, datos con tratamiento técnico y la calidad conocida y apropiada.
La figura 3 representa la estructura del modelo integrado con los elementos ajustados y los niveles de madurez especificado:

Figura 3. Estructura del modelo IQM

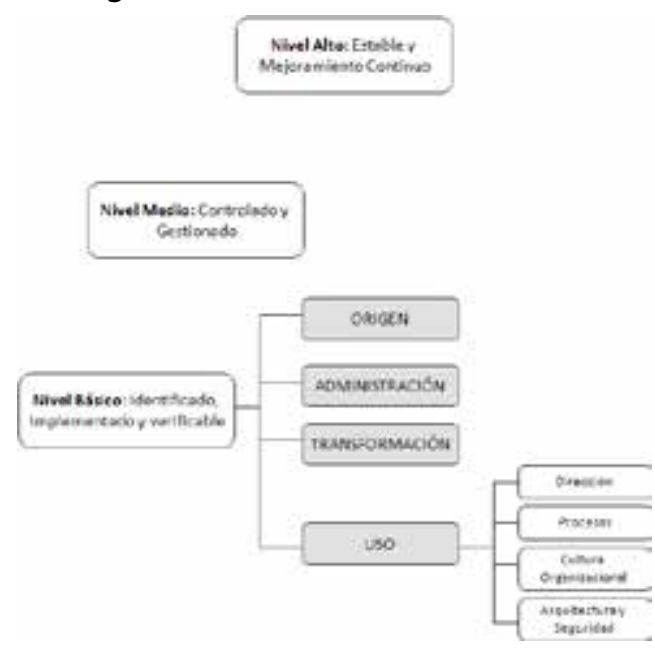

Fuente: Elaboración propia de los autores 


\section{Método para la Valoración del Nivel de Madurez IQM - QDMM}

El sistema de métricas establecido para el modelo, valora las prácticas, las competencias y las capacidades de la empresa en las cuatro perspectivas organizacionales que son consideradas críticas en las organizaciones para la determinación de la calidad de datos.

\section{Áreas de Valoración}

Se establecen dos áreas en las cuales se examinan las prácticas trazadas para la organización con respecto a sus funciones:

- Area Gerencial: esta valoración identifica aspectos de tipo estratégico y de direccionamiento que tienen las empresas hacia la aplicación de prácticas que vayan en beneficio y mejores niveles de garantía de la calidad de los datos en las organizaciones. En esta área se han definido dos perspectivas desde las cuales se realizarán las revisiones correspondientes: Direccionamiento y Cultura Organizacional.

- Area Técnica / Operativa: esta valoración identifica aspectos de tipo técnico y mide la efectividad de las directrices que rigen en la organización los procesos directamente relacionados con la garantía de la calidad de los datos, esencialmente por la interacción de las personas con los sistemas de información que contienen dichos datos. En esta área se han definido dos perspectivas para realizar las revisiones correspondientes: Trazabilidad del dato y Arquitectura e Ingeniería del Dato.

\section{Perspectivas de Direccionamiento del Area Gerencial}

1. Direccionamiento: a partir del análisis y valoración de las prácticas implementadas en la organización, se determina el nivel de preparación sostenible para generar información de calidad para la toma de decisiones eficientes, evidenciado a través de directrices o políticas enunciadas, oficiales, implementadas y auditadas. En esta perspectiva los factores de valoración son: 1) Información estratégica, 2) Planeación estratégica, 3) Políticas de tecnología, 4) Gestión de procesos y 5) Gestión del talento humano.

2. Cultura Organizacional: mide el nivel de incorporación e identidad de las directrices y políticas, en el quehacer del recurso humano que son reconocidas e identificadas como parte de la cultura de una organización en el marco del tratamiento de la información y la calidad de los datos. En esta perspectiva los factores de valoración son: 1) Información estratégica, 2) Planeación estratégica, 3) Políticas tecnología, 4) Gestión de procesos y 5) Gestión del talento humano.

\section{Perspectivas de Direccionamiento del Area Técnica / Operativa}

1. Trazabilidad del Dato: a partir del análisis y valoración de las prácticas implementadas en la organización, se determina el nivel de la presencia de 
buenas prácticas en los procedimientos de validación, medición seguridad y mejoramiento continuo tal que se garanticen la calidad de los datos. En esta perspectiva los factores de valoración son: 1) Origen, 2) Procesos de Administración, 3) Transformación y 4) Uso.

2. Ingeniería del Dato: es el conjunto de características de los insumos utilizados para generar la información estratégica de la organización, compuesta por los datos y los elementos tecnológicos, cuyo nivel de calidad influyen en la capacidad para asegurar la calidad del dato. En esta perspectiva los factores de valoración son: 1) Funcionalidad y 2) Mantenibilidad.

La figura 4 presenta la composición del sistema de métricas a partir de cada uno de sus elementos constitutivos principales (áreas y perspectivas).

Figura 4. Composición del Sistema de Métricas

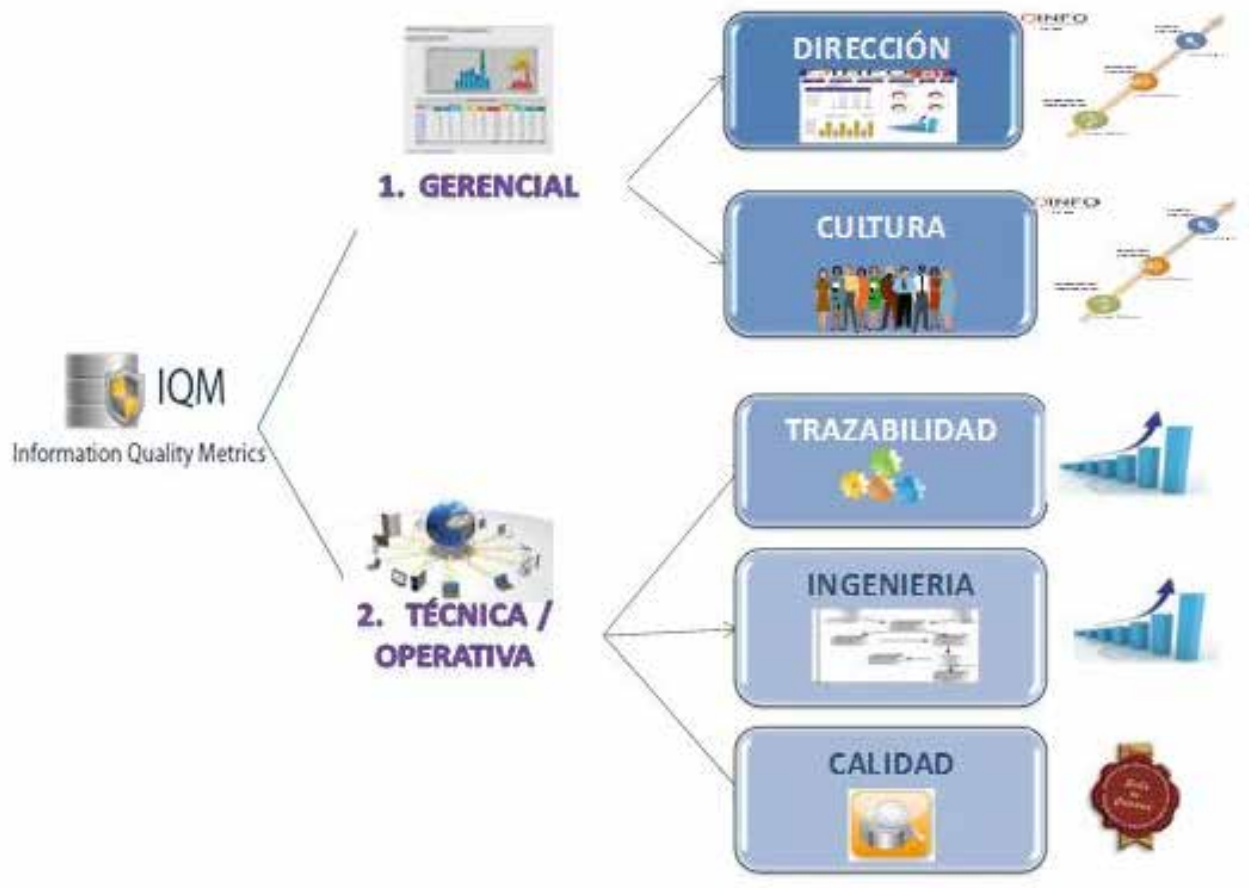

Fuente: Elaboración propia de los autores 


\section{Valoración}

La valoración del nivel de madurez en la calidad de los datos, se determina a partir de la evaluación de cada uno de los factores establecidos, para lo cual se han diseñado diversos instrumentos que dependen del tipo de información que se quiere verificar.

Para los factores asociados a las perspectivas de Direccionamiento y Cultura Organizacional, se han definido una serie de encuestas que incluyen entre 3 y 5 preguntas para valorar cada factor, cada una de las cuales se califica en términos del nivel alcanzado identificado y documentado (Básico), gestionado y controlado (Medio) o Mejorado (Alto); igualmente se han definido aspectos que deben considerarse al momento de realizar el levantamiento de información de dicho factor, así como el análisis de riesgos e impacto en costos para la organización.

Para los factores asociados a las perspectivas de Trazabilidad e Ingeniería del Dato, se han definido los referentes e indicadores que deben examinarse para establecer el nivel en el que se encuentra cada factor.

Finalmente, una vez realizada la valoración se identifica el nivel de madurez en el que se encuentran los datos en los sistemas de información estudiados, se identifican los riesgos presentes y se estiman las pérdidas económicas potenciales por mala calidad en los datos.

\section{Caso de Estudio Descripción \\ de la Organización}

El modelo madurez para la calidad de los datos organizacionales, fue aplicado en una empresa del sector de servicios encargado de la asesoría y capacitación en prevención de riesgos laborales, que cuenta en la actualidad con altos volúmenes de información y documentos y está en una etapa de crecimiento con algunos procesos de negocio y de apoyo automatizados.

\section{Datos básicos de la empresa:}

\begin{tabular}{|c|c|}
\hline Nombre: & Riesgos y Soluciones Ltda. \\
\hline Sede Principal: & Bogotá \\
\hline Otras Sedes: & Oficina en Cúcuta \\
\hline $\begin{array}{l}\text { Representante } \\
\text { Legal: }\end{array}$ & Manuel Antonio Hernández \\
\hline Objeto Social : & $\begin{array}{l}\text { Servicios de Asesoría y } \\
\text { Capacitación en Prevención } \\
\text { de Riesgos Laborales. }\end{array}$ \\
\hline $\begin{array}{l}\text { Año de } \\
\text { constitución: }\end{array}$ & 1997 \\
\hline Página Web: & www.ryshernandez.com \\
\hline $\begin{array}{l}\text { Clientes } \\
\text { principales: }\end{array}$ & Alfa ARP, Colmena ARP \\
\hline $\begin{array}{l}\text { Otros clientes de } \\
\text { servicio: }\end{array}$ & $\begin{array}{l}\text { Más de } 300 \text { empresas } \\
\text { privadas: pequeñas, medianas } \\
\text { y grandes. }\end{array}$ \\
\hline $\begin{array}{l}\text { Número de } \\
\text { empleados: }\end{array}$ & 43 \\
\hline
\end{tabular}

La misión de la empresa es:

"Contribuir en la prevención, protección y atención a los trabajadores, con un cambio de actitud hacia la prevención, mediante el liderazgo en la atención integral de Salud Ocupacional, con un equipo humano altamente calificado y comprometido, basado en los principios de ética, eficiencia y participación que conduzcan a la organización a su crecimiento y sostenibilidad en el tiempo" 


\section{Proceso seleccionados}

Los procesos seleccionados sobre los cuales se realizó el piloto de prueba del método y la herramienta de software asocia$\mathrm{da}$, fueron los siguientes:

- Proceso de Recepción de solicitudes de trabajo: proceso a través del cual ingresa una solicitud de servicio a la organización y termina con la creación de una orden de trabajo interna.

- Proceso de Ordenes de Servicio: es el proceso que parte de las órdenes de servicio aprobadas, en el mismo se asignan los profesionales que realizarán las actividades respectivas y se organiza el cronograma, una vez realizada la actividad se verifica la documentación y se confirma la contabilidad para que proceda a la facturación.

- Proceso de Facturación: es el proceso a través del cual se registra la información en el software contable, donde se generan las facturas y el proceso de cobro de la empresa.

\section{Actividades ejecutadas}

Se valoraron las perspectivas de Direccionamiento y Cultura Organizacional (nivel de negocios de acuerdo con la metodología) para el Modelo de Madurez, aplicando sus conceptos y evidenciando su efectividad en los resultados.

Se realizó el levantamiento de la trazabilidad de datos para los procesos definidos, generando un resultado de mucha utilidad para la empresa por la visión macro y mapeo que se elabora sobre el comportamiento de los datos en la organización, calificado como innovador.

Se valoró el Ciclo de Vida del Dato (CVD) conforme lo establece el Método, elaborando la métrica o estado actual de la organización en cada uno de los factores que componen el ciclo: origen, administración, transformación y uso de los datos.

Se realizó la valoración de aspectos de ingeniería del dato, en los cuales se aplicó una herramienta de software la cual genera a través de sus resultados las evidencias y confirmación de riesgos que resultan del levantamiento de las perspectivas del negocio evaluadas.

Se establecieron evidencias a través de la aplicación de análisis de calidad de dato con dos bases de datos y tres tablas de misión crítica, que orientan a la empresa en las principales acciones correctivas o de mejora a implementar.

\section{Herramientas de software aplicado al pilloto}

Como herramienta de software, se empleó la desarrollada para la metodología, la cual ayuda a identificar el nivel en el que se encuentra la empresa respecto a la calidad de los datos (básico, medio o alto) y consta de cuatro módulos:

1. Administrador: módulo en el cual se parametrizan las tablas del sistema, los usuarios, las empresas y se definen las estructuras de los archivos planos que se requieren procesar.

2. Línea base: módulo en el cual se ingresan todos los datos relacionados con encuestas que se aplican a nivel de Dirección y Cultura Organizacional, y a nivel de Procesos y Arquitectura de 
Datos. Igualmente se incluyen los archivos planos sobre los cuales se quieren generar indicadores y diagnóstico de reparación de datos básicos.

3. Análisis: en este módulo se realizan los procesos de análisis y se generan las métricas para los archivos que se requieran.

4. Informes: módulo en el cual se presentan los resultados a nivel de encuestas, diagnóstico y recomendaciones.

\section{Resultados}

Los principales resultados obtenidos con la aplicación del modelo son los siguientes:

1. Valiosa visión global de la calidad de los datos. Se evidenció la ventaja de hacer una observación general y no solamente técnica del proceso piloto.

2. Evidencia de prácticas erradas de la empresa. En la medida que se avanza en el proceso, se logra evidenciar puntos que la organización por sí sola no evidenciaba y que se identifican como "escenarios relevantes" para el tema de la calidad de los sistema de información.

3. Evidencia fallas en seguridad de los datos. A partir de algunas evaluaciones, se identifican las vulnerabilidades que se tienen en la empresa y que producen riesgos de pérdida de información, y se establecen nuevos niveles de valoración de algunos datos realmente críticos para el negocio.

4. Integración de procesos estratégicos con procesos operativos y técnicos en la valoración de la empresa, bajo el lente de la calidad de los datos.

\section{Conclusiones}

Es relevante tener un modelo aplicado a los datos (que son de base tecnológica) que contenga un lenguaje de negocios, teniendo en cuenta técnicas y prácticas que domina la empresa e incluya a las personas de la organización que están vinculadas con la Trazabilidad de los datos, es decir, con todo el sistema de información de la empresa.

Es crítico adelantar un análisis del sector y el entorno de la empresa para tener claridad sobre la importancia estratégica de los datos, con respecto a su modelo de negocio.

Las perspectivas de Cultura Organizacional y Trazabilidad del dato, establecen un diferenciador con otras técnicas o métodos de valoración de calidad del dato, haciendo más efectiva la visualización de vulnerabilidades y proponiendo soluciones de espectro más amplio al cumplimiento de las metas.

El tipo de análisis y de documentación producida, tiene un enfoque para diferentes escenarios y actores de la organización, permitiendo visualizar aspectos de orden estratégico y de orden táctico.

Para las organizaciones, tener un plan de mejoras simple pero efectivo, dará posibilidades de hacer realidad en cortos plazos, eficiencias que desde los datos y la información, redunden en beneficios tangibles. Este aspecto se consolida muy bien con la aplicación de métricas bajo un modelo de madurez y con un enfoque sistemático que ofrece IQM. 


\section{Referencias}

5. Allen, M.D., Carter, S., Aiken, P., Cyrus, M.K., Wade, K., McCormac, S. (2003). Extracting Data from Free Text Fields: Assuring Data Quality for ERP Implementation. Proceedings of the Eighth International Conference on Information Quality, pp.319-330.

6. Amicis, F. D., Barone, D., Batini, C. (2006). An Analytical Framework to Analyze Dependencies among Data Quality Dimensions. En: Talburt, J., Pierce, E., Wu, N. and Campbell, T. (Eds), Proceedings of the 11th International Conference on Information Quality, pp.369-383.

7. Batini, C., Barone, D., Mastrella, M., Maurino, A., Ruffini, C. (2007). A Framework and a Methodology for Data Quality Assessment and Monitoring. Proceedings of 12th International Conference on Information Quality. Ciudad.

8. Eckerson, W. (2002). Data Warehousing Special Report: Data quality and the bottom line. Ciudad: editorial.

9. ESI Center - SINERTIC. (2011). Metodología del Servicio IQM.

Io. Even. A, Shankaranarayanan, G., Berger, P. D. (2005). Profit Maximization with Data Management Systems. Proceedings of the International Conference on Information Systems. Las Vegas.

I I. Führing, P., Naumann, F. (2007). Emergent Data Quality Annotation and Visualization. Proceedings of the Twelth International Conference on Information Quality (ICIQ), pp.424-430.

I 2. Heinrich, B., Kaiser, M., Klier, M. (2007). DQ metrics: a novel approach to quantify currency and its application in CRM. Proceedings of the 12th
International Conference on Information Quality (ICIQ 07), pp.431-445.

I3. Hipp, J., Müller, M., Hohendorff, J., Naumann, F. (2007). Rule-Based Measurement of Data Quality In Nominal Data. Proceedings of the 12th International Conference on Information Quality (ICIQ 07), pp.364-378.

I4. Kerr, K., Norris, T. (2004). The development of a healthcare data quality framework and strategy. Ninth International Conference on Information Quality (IQ 2004), pp.218-233.

I 5. Leonowich-Graham, P., Willshire, M.J. (2003). A Data Quality Framework for Small Businesses. Proceedings of the 8th International Conference on Information Quality, pp. 239-244.

I6. Long, J. A., Seko, C. E. (2002). A New Method for Database Data Quality Evaluation at the Canadian Institute for Health Information (CIHI). Proceedings of the International Conference on Information Quality (IQ), pp. 238-250.

17. Nyaboga, A. B., Nwuara, M. F. (2009). Strategies for Gaining Competitive Advantage in A Dynamic Environment Thru Data Quality. International of Management \& Information System. 13(1): 13-22.

I8. Oliveira, P., Rodrigues, M.F., Henriques, P.R. (2006). An Ontology-Based Approach for Data Cleaning. Proceedings of the $11^{\text {th }}$ International Conference of Information Quality (ICIQ 2006), pp.307-320.

I9. Savchenko, S. (2003). Automating Objective Data Quality Assessment. Proceedings of the 8th International Conference on Information Quality, pp. 353-364.

20. Shankar, G., Watts, S. (2003). A Relevant, Believable Approach for Data Quality Assessment. Proceedings of the 
8th International Conference on Information Quality, pp.178-189.

2 I. SINERTIC. (2009). Metodología de la Línea de Base del Servicio QINFO.

22. Wang, H., Wang, S. (2007). Mining purchasing sequence data for online customer segmentation. International Journal of services operations and informatics, 2(4), 382 - 390.

23. 